\title{
Analysis of Resonant Tunnelling Diode Oscillators under Optical Modulation
}

\author{
J. S. Tavares ${ }^{1,2}$, L. M. Pessoa ${ }^{1}$, J. M. L. Figueiredo ${ }^{3}$, H. M. Salgado ${ }^{1,2}$ \\ ${ }^{I}$ INESC TEC, Porto, Portugal \\ ${ }^{2}$ Faculty of Engineering, University of Porto, Porto, Portugal \\ ${ }^{3}$ Departamento de Física, CEOT, Universidade do Algarve, Portugal \\ e-mail: joana.tavares@fe.up.pt
}

\begin{abstract}
In this paper, we investigate the optical modulation characteristics of a resonant tunnelling diode (RTD) oscillator. This preliminary work on the first RTD-PD oscillators with an optical window available from the iBROW project demonstrate that this device can effectively be used to accomplish amplitude and frequency modulation with light injection.
\end{abstract}

Keywords: resonant tunnelling diode (RTD), optical modulation, oscillator.

\section{INTRODUCTION}

The demand for broadband content and services using both wired and wireless access has been growing steadily. Current market trends predict that wireless data-rates of multiple tens of Gbps will be required by the year 2020 . With the aim of devising new hardware solutions to address this challenge, the iBROW project has been leveraging Resonant Tunnelling Diode (RTD) based technology to achieve a unified technology that can be integrated into both ends of a wireless link, namely consumer portable devices and fibre-optic supported base-stations (Figure 1). Two types of RTDs are required to address the iBROW concept, an all-electronic RTD suitable for low cost, and low power portable devices, as well as an opto-electronic RTD, suitable for fibre-optic supported base-stations, with the optical-to-wireless interface consisting of an RTD incorporating a light absorption region, forming a RTD photo-detector (RTD-PD), and the wireless-to-optical interface realized through the hybrid integration of an RTDPD with a laser diode (RTD-PD-LD) [1].

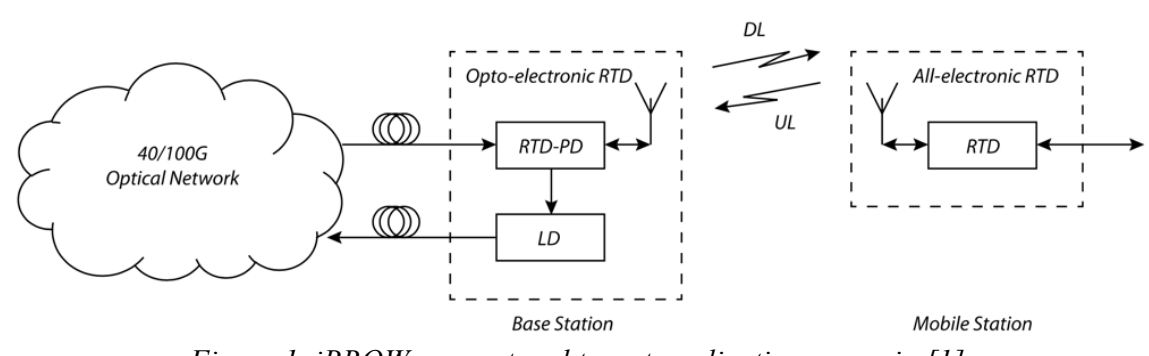

Figure 1. iBROW concept and target application scenario [1]

Double barrier quantum well (DBQW) RTD structures for photo-detection emerged in the last decades as devices with high responsivities (also denoted as sensitivity [2]), high gain-bandwidth-efficiency products, low switching energies and low voltage operation characteristics [3]. To improve photo-detection capabilities of RTDs a photoconductive layer is generally added to the epi-layer structure [4]. An RTD incorporating a photo-conductive region can operate as a photo-detector, the RTD-PD which, when interfaced with an antenna, can radiate the wireless signal generated by its photocurrent. The RTD-PD corresponds to an optoelectronic device with built-in amplification, which can convert either a baseband modulated or RF modulated subcarrier optical signal, impinging on its optical window, to a high frequency RF carrier.

This paper presents preliminary results on the optical response of the first RTD-PD oscillators available from the iBROW project. Our results show that this device can be used to employ amplitude modulation of light.

\section{DEVICE AND EXPERIMENTAL SETUP}

The RTD is a unipolar two terminal semiconductor with a N-shaped current-voltage (I-V) characteristic at room temperature, exhibiting voltage controlled negative differential conductance (NDC) regions which provides electrical gain allowing the implementation of very simple high frequency electrical oscillators and a myriad of other electronic and optoelectronic devices with new functionalities [5]. The RTD photodetector (RTD-PD) object of study here corresponds to a vertical stacking of nanometric scale semiconductor epi-layers consisting of an undoped indium gallium arsenide (InGaAs) layer sandwiched between two aluminium arsenide (AlAs) barrier layers, forming a double barrier quantum well (DBQW), surrounded by low doped InGaAs spacer epi-layers which act as light absorbing regions where photo-generated electron-holes are created upon incident light. The final 
structure is completed with very high-doped InGaAs layers for ohmic contact formation. The structure is normally realized on semi-insulating (SI) InP substrates, but other substrates are possible such as SiGe or Si.

The RTD oscillator circuit considered here employs two RTD-PDs in parallel connected to a shorted $50 \Omega$ coplanar waveguide $(\mathrm{CPW})$ line which provides the inductance required to be resonant with RTD-PDs selfcapacitance for a given targeted oscillation frequency (here $\sim 25 \mathrm{GHz}$ ), as shown in Figure 2. The individual RTDPD device size is $10 \times 10 \mu \mathrm{m}^{2}$, with an optical window of $10 \mu \mathrm{m}$ by $5 \mu \mathrm{m}$, which corresponds to half of the device mesa size. Figure 3 presents the experimental setup diagram. The RTD-PD oscillator was biased by landing an RF probe on the CPW on the shorted side (oscillator DC port). The tip of the fibre of a pigtailed DFB laser was also aligned with RTD-PD using a microscope. The laser was in turn directly modulated by a single subcarrier frequency generated by the vector signal generator (VSG). The light signal was transmitted to the RTD-PD using a cleaved fibre aligned on top of the optical window of the device, at an angle of approximately $20^{\circ}$ of the surface normal. Another RF probe was landed on the oscillator RF port (left side of Figure 2) and the received signals were observed on the spectrum analyser. Furthermore, a GPIB connection was used to control the power supply and the spectrum analyser, to easily register the results. This setup allows us to experimentally characterise the oscillator electrical and optical/optoelectronic response when light is injected into the RTD-PD.

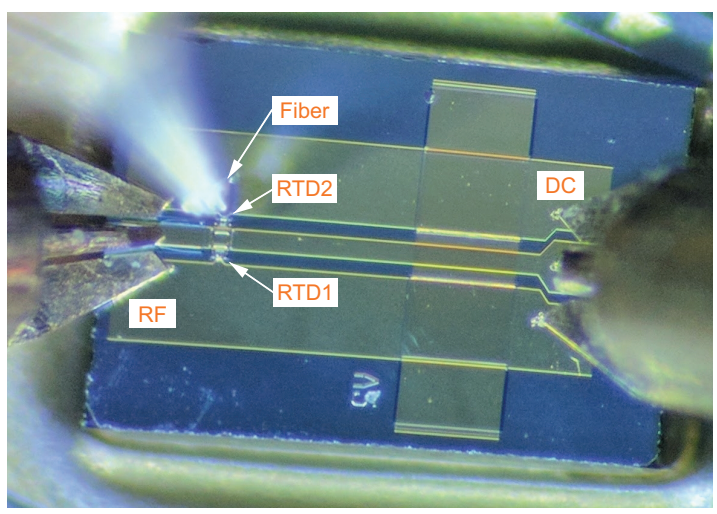

Figure 2. Photograph of the oscillator employing 2 RTD devices, the optical fibre (over the optical window) and $R F$ (left) and DC (right) probes.

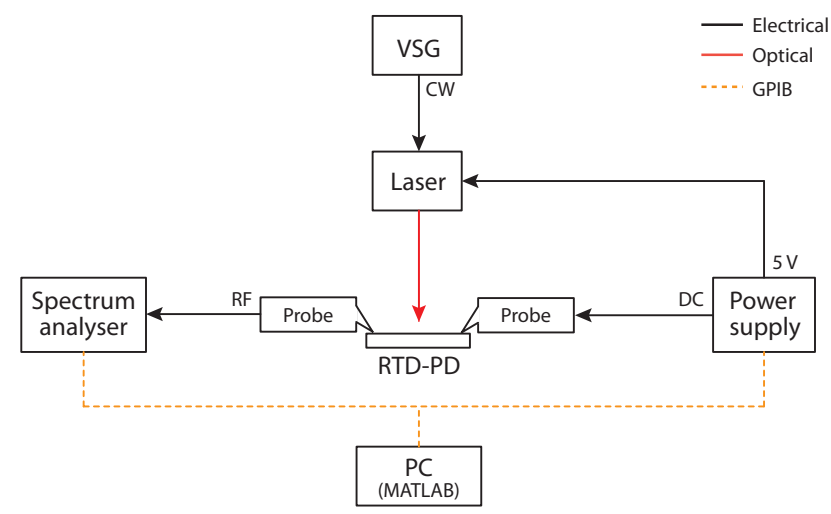

Figure 3. Experimental setup diagram.

\section{EXPERIMENTAL RESULTS}

First, we obtained the I-V curve of the RTD-PD circuit in three different situations: dark (no light injected), CW optical carrier only and modulated optical carrier, presented in Figure 4. The two slopes are signatures of the two RTD-PDs NDC (negative differential conductance) regions. The inset clearly shows that, in the presence of light, the I-V curve shifts to the right and the current increases, which was expected since a significant portion of the injected light is being absorbed in the emitter side of the illuminated device.

Figure 5 presents the observed full power spectrum when the laser was directly modulated by a single subcarrier at $500 \mathrm{MHz}$, with the RTD-PD oscillating at approximately $25.5 \mathrm{GHz}$ and the sidebands located at $500 \mathrm{MHz}$ from the carrier.

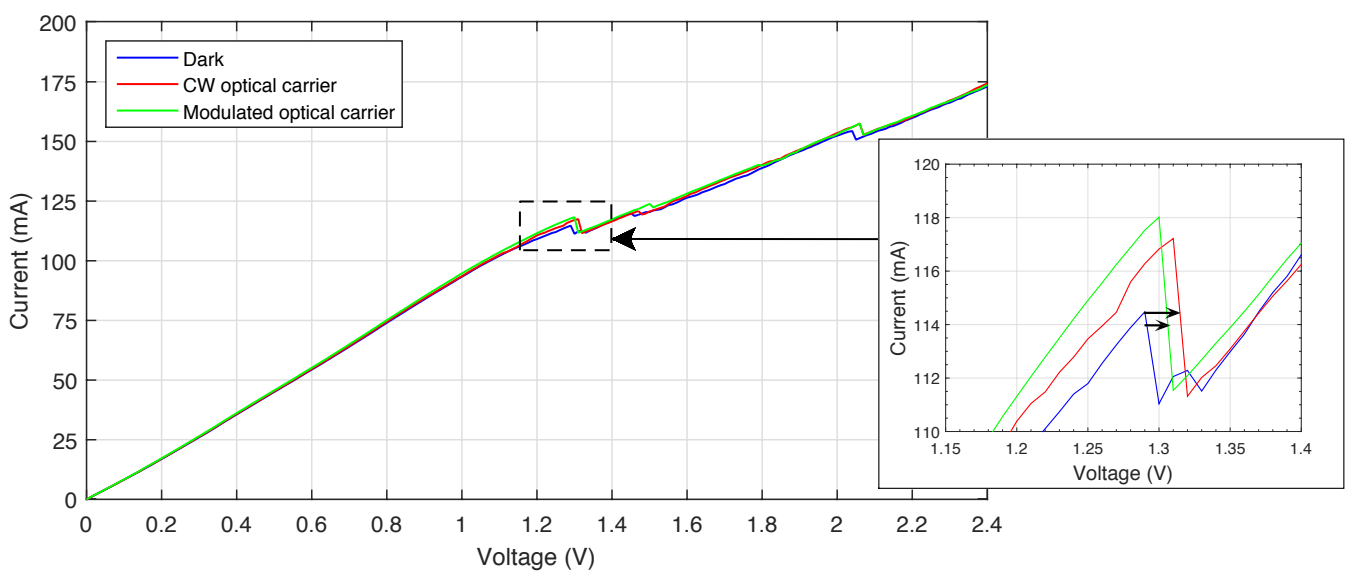

Figure 4. Measured I-V characteristic curve of the oscillator. Inset: amplification showing the I-V curve shifting to the right in the presence of light. 


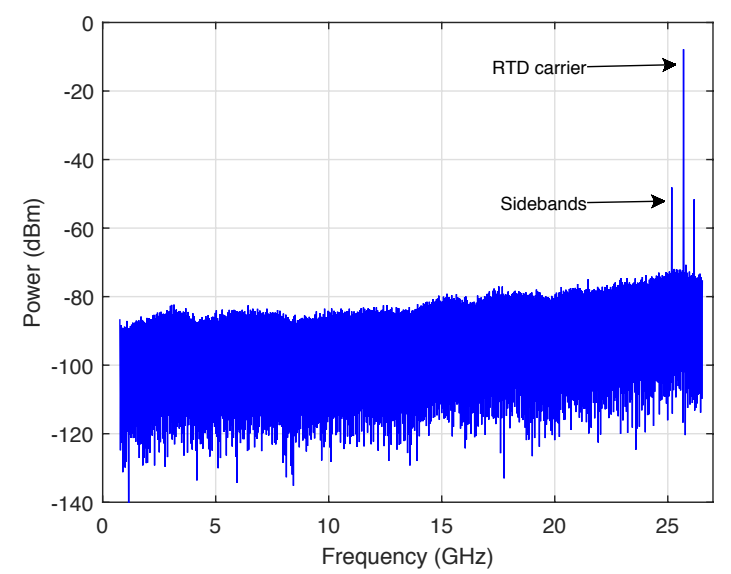

Figure 5. Power spectrum showing the RTD oscillating at $25.5 \mathrm{GHz}$ and the sidebands at $500 \mathrm{MHz}$ from the carrier.

The next experiment was carried out to measure the oscillator operating frequency and output power as a function of the DC bias voltage. We performed this experiment with and without light injection. The oscillation frequency and the corresponding generated power increases smoothly with the bias voltage, reaching a maximum within the NDC region, as shown in Fig. 6 and Fig. 7, respectively. In the presence of light the overall behaviour as a function of the bias is the same but the presence of light leads to an increase of the generated power throughout all the NDC region and to a decrease of the oscillating frequency. The power increase with the optical injection is owed to the increase of the current induced by the presence of light. In turn, the frequency decrease may be due to the increase of the capacity as a result of the generation of electron-hole pairs in the depletion region (collector region next to the second barrier). This behaviour was expected and can be further explored to employ amplitude or frequency modulation.

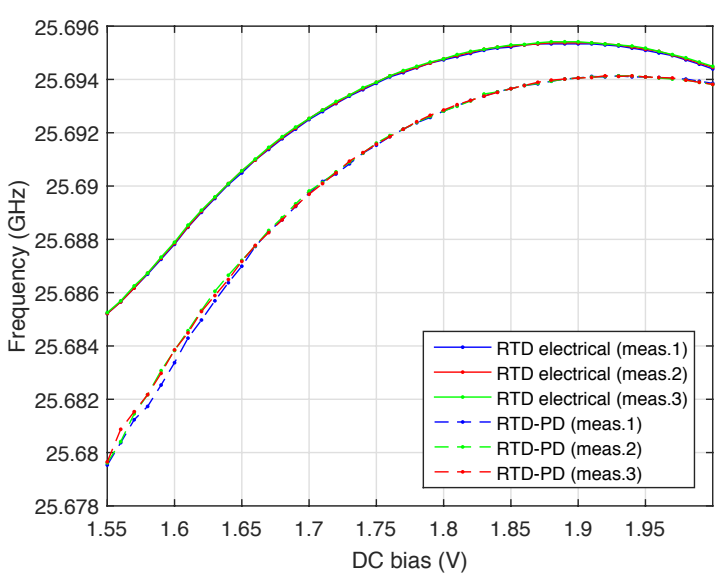

Figure 6. Oscillator frequency vs. DC bias voltage.

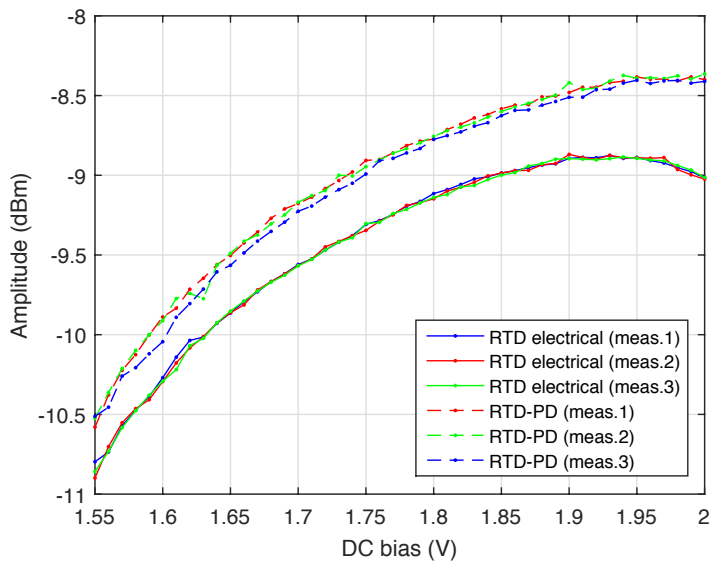

Figure 7. Oscillator output power vs. DC bias voltage.

The baseband photodetected power from the RF probe was observed for two different frequencies. The best result was obtained with $1.3 \mathrm{~V}$ of DC bias voltage, showing a photodetected power of $-66 \mathrm{dBm}$ at $500 \mathrm{MHz}$ and $-72 \mathrm{dBm}$ at $1 \mathrm{GHz}$ (Figure 8). These results are being further improved with a better alignment and using a lensed fibre. Initial results points to improvements as high as $40 \mathrm{~dB}$.

Next, we performed a frequency sweep for two different DC bias voltages $(1.8 \mathrm{~V}$ and $2.0 \mathrm{~V})$ and measured the power of the lower side band, as shown in Figure 9. We present only the lower sideband due to the spectrum analyser bandwidth limitation of $26.5 \mathrm{GHz}$. This result shows a lower sideband with up to $6 \mathrm{GHz}$ modulation frequency. We observed that the RTD-PD carrier power increases with DC bias, but the optical modulation sideband power decreases.

Lastly, we obtained the lower sideband peak power and frequency as a function of DC bias voltage, for different modulation frequencies. Figure 10 shows that the output power decreases with the frequency and with DC bias.

We expect to be able to reach values superior to $-40 \mathrm{dBm}$ in this frequency range as the result of improvement of the epi-layer design and of the light coupling efficiency through better alignment and by using a lensed fibre. 


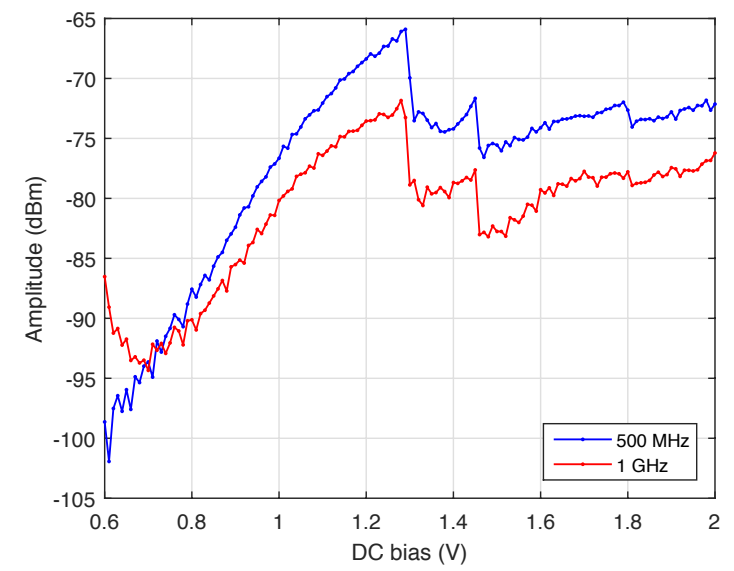

Figure 8. Baseband photodetected power at $500 \mathrm{MHz}$ and $1 \mathrm{GHz}$ vs. DC bias voltage.

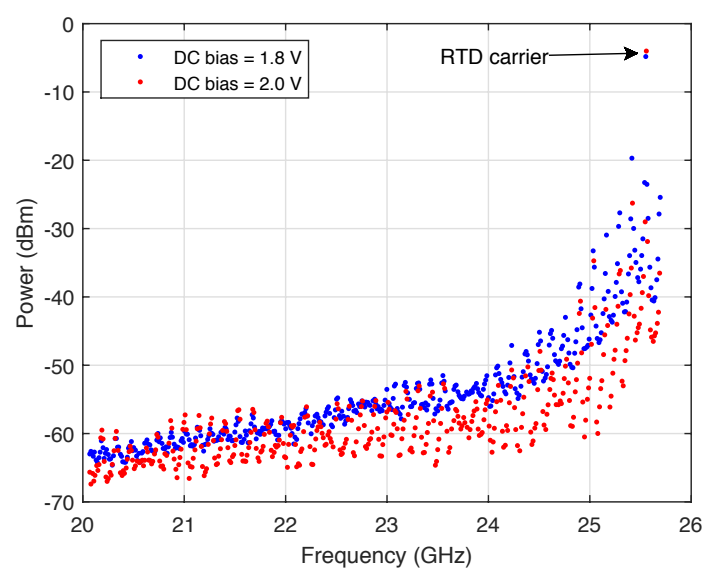

Figure 9. Frequency sweep for two different DC bias points.

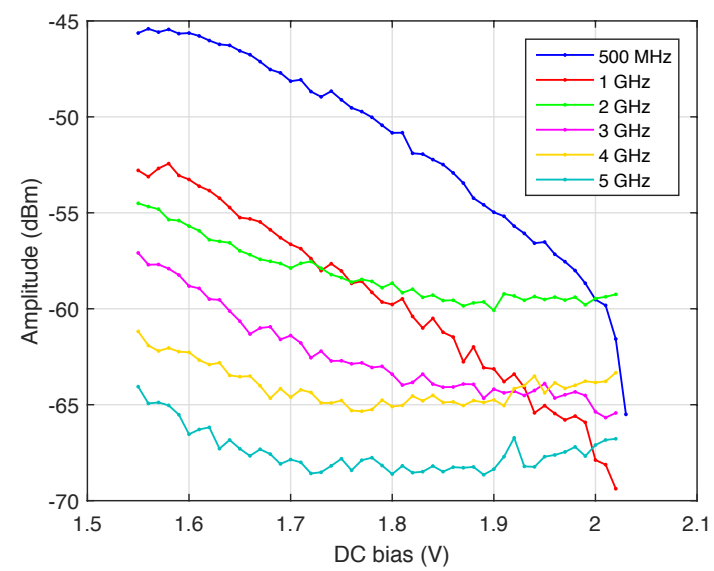

Figure 10. Low sideband power as a function of the DC bias voltage.

\section{CONCLUSIONS}

In this work, we experimentally evaluated the optical response of the first RTD-PD oscillators available from the iBROW project. This preliminary study shows relevant results that demonstrate the feasibility of amplitude and frequency modulation by light injection. Moreover, we expect to achieve higher output power by improving the RTD design in the next iterations and by improving the light injection technique.

\section{ACKNOWLEDGEMENTS}

The authors would like to thank Edward Wasige, Scott Watson, Anthony Kelly, Jue Wang and Abdullah AlKhalidi at the University of Glasgow for providing the RTD and for the technical support. This work was supported by the iBROW project (grant agreement no. 645369), supported by the European Commission under the Horizon 2020 Programme, and by FCT (Fundação para a Ciência e a Tecnologia, Portugal), through the research grant $\mathrm{PD} / \mathrm{BD} / 113821 / 2015$.

\section{REFERENCES}

[1] L. Pessoa, B. Peng, J. Wang, L. Sanchez, A. Al-Kahlidi, E. Wasige, A. Liu, H. Cantu, I. Oxtoby, B. Napier, iBROW-innovative ultra-BROadband ubiquitous wireless communications through terahertz transceivers, IEEE COMSOC MMTC Communications-Frontiers, 11, p. 12-17 (2016)

[2] A. Pfenning, F. Hartmann, F. Langer, M. Kamp, S. Höfling, and L. Worschech, Sensitivity of resonant tunneling diode photodetectors, Nanotechnology 27 (2016) 355202 (9pp)

[3] T. S. Moise,Y.-C. Kao, C. L. Goldsmith, C. L. Schow, and J. C. Campbell, High-Speed ResonantTunneling Photodetectors with Low-Switching Energy, IEEE Phot. Techn. Letters, 9, p. 803-805 (1997).

[4] B. Romeira, L. Pessoa, H. M. Salgado, C. N. Ironside, and J. M. L. Figueiredo, Photo-detectors integrated with resonant tunneling diodes, Sensors, vol. 13 (7), pp. 9464-9482, Julho 2013.

[5] J. Figueiredo, B. Romeira, T. Slight, C. Ironside, Advances in optical and photonic devices, InTech2010) 